\title{
Molecular analyses in wheat and Aegilops tauschii reveals a new orthologue of the leaf rust resistance gene Lr19 on 7DL chromosome of Ae. tauschii
}

\section{Zahra Pourkhorshid}

Shiraz University https://orcid.org/0000-0001-6336-4432

Ali Dadkhodaie ( $\sim$ dadkhodaie@shirazu.ac.ir)

Shiraz University https://orcid.org/0000-0001-7824-9162

Roohollah Shamloo-Dashtpagerdi

Higher Education Center of Eghlid https://orcid.org/0000-0001-6449-305X

\section{Research Article}

Keywords: Aegilops tauschii, Leaf rust, Lr19, Molecular markers, NBS-LRR, O-methyltransferase

Posted Date: December 1st, 2021

DOI: https://doi.org/10.21203/rs.3.rs-1080624/v1

License: (9) (1) This work is licensed under a Creative Commons Attribution 4.0 International License. Read Full License

Version of Record: A version of this preprint was published at Journal of Phytopathology on January 30th, 2022. See the published version at https://doi.org/10.1111/jph.13077. 


\section{Abstract}

Leaf rust is one of the most devastating wheat diseases worldwide, to which many resistance genes have been successfully introgressed from wheat wild relatives. Though the Thinopyrum ponticum-derived leaf rust resistance gene $L r 19$, is widely effective worldwide and previous studies have shown its likely presence in Aegilops tauschii, no thorough investigation has been conducted to confirm this. The present study aimed to examine the presence of Lr19 in Ae. tauschii using a collection of molecular and bioinformatic analysis. Accordingly, the Thatcher line was used as susceptible, and a Thatcher $+L r 19$ (TCLr19) and Agatha were used as resistant lines. CDHLQ pathotyping coupled with DNA markers genotyping verified the presence of an Lr19 orthologue on Ae. tauschii 7DL (AtLr19). Sequencing of the GB marker products from Ae. tauschii and TcLr19 showed 99\% homology in these fragments, confirming phenotyping and genotyping results. Both isolated segments were matched to a putative melatonin biosynthesis gene, namely 0 -methyltransferase-2 (OMT2) mapped to 7DL, with $100 \%$ identity. A hierarchical gene network was reconstructed using all identified putative genes within a genomic region containing $2.5 \mathrm{cM}$ upstream and downstream of the OMT2 gene. Results indicated that several numbers of important biotic stress-responsive genes such as RPM1, RGA2, TRIUR3, BURP12, and myosin-11, were located downstream of melatonin as a master regulator molecule through the OMT2 node. To our knowledge, this is the first report of finding an orthologue for Lr19 in Ae. tauschii, which provides insights into the possible regulatory route of $L R 19$.

\section{Introduction}

Wheat production has increased from 235 million tons in 1961 to approximately 766 million tons in 2019 (FAO, 2021), enough to feed the current world population which is expected to reach 10 billion in 2050 . This necessitates further crop production, especially wheat as a major food source. However, this crop faces multiple biotic and abiotic challenges that pose a major concern for increasing production (Mondal et al., 2016). Globally, the three rusts i.e. leaf rust (Puccinia triticina, $P t)$, stripe rust (P. striiformis f. sp. tritici, Pst) and stem rust (P. graminis f. sp. tritici, Pgt), are the most devastating wheat diseases which cause substantial yield losses (Huerta-Espino et al., 2011; Pardey et al., 2013; Wellings, 2011).

Among the existing control approaches of rust, incorporating disease resistance genes (R genes) into wheat cultivars is the most efficient and eco-friendly strategy where new and effective resistance genes are deployed. Though many rust resistance genes have originated from hexaploid wheat, the primary and secondary gene pools accommodate new functional variants for agronomically relevant genes (Feuillet et al., 2008) and hence, wheat wild relatives such as Aegilops, Dasypurum, Thinopyrum and Secale have contributed to numerous $\mathrm{R}$ genes (Mondal et al., 2016). This highlights the fact that disease resistance breeding in wheat has greatly depended on these genetic resources since its inception.

Rust resistance genes are postulated by comparing infection type (IT) patterns of tested genotypes with those of differential cultivars/lines inoculated by an array of pathotypes where the presence/absence of a resistance gene is decided based on low or high ITs. In some cases, pedigree information are used to 
confirm the results of gene postulation (Randhawa et al., 2016). Yet, this approach requires a wellcharacterized collection of pathotypes. Moreover, the results need validation through genetic analysis and/or using molecular markers.

Traditional and molecular marker technology approaches have been successfully utilized to introgress alien chromosome segments carrying resistance genes for all three rusts (Mondal et al., 2016). A functional molecular marker should give highly repeatable results in different gene pools. In the context of genetic investigation, molecular markers have a proper selection intensity and permit the screening of resistance genes in wheat collections (Toth et al., 2019) as well as tracking them in breeding lines or gene pyramiding. Nowadays, bioinformatics tools are being used to search genes within the target genomes and elucidate their functions (Aslam et al., 2017). These new developments have assisted in a comprehensive understanding of the relationship between structural and functional gene organization during evolution and have revealed that the organization of genes has remained more sustained and conserved than it was previously perceived (Caetano-Anolles, 2005). Therefore, these tools can be used to assign a function to a gene and a protein by studying similarities between different sequences (Stormo, 2000).

To date, more than 80 leaf rust resistance genes have been characterized and formally designated (McIntosh et al., 2019). Despite the success of gene deployment in averting rust infection, single resistance genes are rapidly overcome by variations in the $P$. triticina $(P t)$ populations. One of the few widely effective genes conferring resistance to leaf rust; $L r 19$, was transferred from the wild relative Thinopyrum ponticum, a diploid genus belonging to the poaceae family, into wheat where it is located on the long arm of the wheat chromosome 7D (Sarma and Knott, 1966). This gene produces a hypersensitive response (Plotnikova, 2008) and rust surveys have shown its effectiveness over time and space (McCallum et al., 2021). Though Lr19 was originated from Th. ponticum, Aliakbari et al. (2020) observed ITs similar to that of Thatcher+Lr19 (TcLr19) and Lr19-corresponding band in few Aegilops tauschii genotypes. Yet, no investigation has performed to confirm this. Therefore, this study investigated the phenotype and genotype of Lr19-carrying/lacking wheat and Ae. tauschii genotypes. Moreover, the functionality of molecular markers associated with $L r 19$ was verified, and sequencing and bioinformatics studies compared this gene in wheat and Ae. tauschii genotypes. To our knowledge, this is the first report of investigating Lr19 in Ae. tauschii.

\section{Material And Methods}

\subsection{Plant Templates}

Two Ae. tauschii accessions SU442 and SU444 displaying the same resistance response to $P$. triticina as the Lr19 carrying genotypes were selected from a larger collection of wheat wild relatives (Aliakbari et al., 2020). Accordingly, for more precise investigation, TcLr19 (RL6040) and Agatha (Agrus/6xThatcher) as positive controls, and the susceptible accession SU350, and Thatcher as the negative control were 
included in the experiment. Ten seeds from each genotype were planted in $10 \mathrm{~cm}$ diameter pots under greenhouse conditions of $18 \pm 2^{\circ} \mathrm{C}$.

\subsection{Puccinia triticina inoculation and disease assessment}

The Pt pathotype CDHLQ (virulent 3a, 3bg, 11, 14b, 24, 30, B) was used to inoculate plants. Twelve days after sowing, two-leaved seedlings were inoculated with fresh urediniospores mixed with talcum powder in a 1:4 ratio on upper leaf surface using a fine paintbrush. Then, the inoculated plants were incubated overnight in a dark chamber under $100 \%$ humidity at $18^{\circ} \mathrm{C}$, after which were moved into microclimates kept at $20 \pm 2^{\circ} \mathrm{C}$. The ITs were recorded 12 days post inoculation (dpi) using the method described by McIntosh (1995), where plants with ITs of " 0 ", “;", "1" and " 2 " were considered resistant while those with ITs of " 2 ", " 3 " or higher were regarded as susceptible.

\subsection{Molecular experiments}

Four primer pairs including the STS marker GB, the SSR marker Xwmc221, and two SCAR markers SCS123 and SCS253 (Table 1) reported as associated with Lr19, were used to investigate the presence/absence of this gene in the tested genotypes.

Table 1

Different primer pairs reported as linked to the leaf rust resistance gene, $L r 19$, were used to screen several Ae. tauschii and wheat genotypes for presence/absence of this gene

\begin{tabular}{|c|c|c|c|c|c|}
\hline $\begin{array}{l}\text { Primer } \\
\text { pair }\end{array}$ & $\begin{array}{l}\text { Marker } \\
\text { type }\end{array}$ & Sequence & $\begin{array}{l}\text { Band } \\
\text { size (bp) }\end{array}$ & $\begin{array}{l}\text { Annealing } \\
\text { temperature } \\
\left({ }^{\circ} \mathrm{C}\right)\end{array}$ & Reference \\
\hline \multirow[t]{2}{*}{$w m c 221$} & \multirow[t]{2}{*}{ SSR } & F: ACGATAATGCAGCGGGGAAT & \multirow[t]{2}{*}{$200 / 220$} & \multirow[t]{2}{*}{61.5} & \multirow{6}{*}{$\begin{array}{l}\text { (Gupta et } \\
\text { al., 2006) }\end{array}$} \\
\hline & & R: GCTGGGATCAAGGGATCAAT & & & \\
\hline \multirow[t]{2}{*}{ SCS123 } & \multirow[t]{2}{*}{ SCAR } & F: CCTGATCACCAATGACGATT & \multirow[t]{2}{*}{680} & \multirow[t]{2}{*}{60} & \\
\hline & & R: CCTGATCACCTTGCTACAGA & & & \\
\hline \multirow[t]{2}{*}{ SCS253 } & \multirow[t]{2}{*}{ SCAR } & F: GCTGGTTCCACAAAGCAAA & \multirow[t]{2}{*}{730} & \multirow[t]{2}{*}{58} & \\
\hline & & R:GGCTGGTTCCTTAGATAGGTG & & & \\
\hline \multirow[t]{2}{*}{$G B$} & \multirow[t]{2}{*}{ STS } & F: CATCCTTGGGGACCTC & \multirow[t]{2}{*}{130} & \multirow[t]{2}{*}{59.8} & \multirow{2}{*}{$\begin{array}{l}\text { (Prins et } \\
\text { al,. 2001) }\end{array}$} \\
\hline & & R: CCAGCTCGCATACATCCA & & & \\
\hline
\end{tabular}

Genomic DNA was extracted from clean and fresh leaves of each plant using the CTAB method (Yu et al., 2017). A Nanodrop ND-1000 (Thermo Fisher Scientific, US) was used to measure DNA quantity and quality. The working solutions for both genomic DNA and primers were prepared in $200 \mu$ volumes at a concentration of $50 \mathrm{ng} \mathrm{\mu l}^{-1}$. Polymerase chain reaction (PCR) was conducted in a final volume of $20 \mu \mathrm{l}$

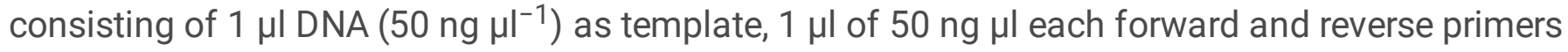
(Metabion, Germany), $5 \mu \mathrm{l}$ Taq DNA Polymerase 2x Master Mix Red ( $5 \mathrm{U} \mathrm{\mu l}^{-1}$, Ampliqon, Denmark), and 12 
$\mu \mathrm{lddH_{2 }}$ O. The PCR conditions were $94^{\circ} \mathrm{C}$ for 5 minutes, followed by 35 cycles of $94^{\circ} \mathrm{C}$ for one minute, 58 $61.5^{\circ} \mathrm{C}$ for one minute depending on the primer pair, $72^{\circ} \mathrm{C}$ for one minute, and 10 minutes at $72^{\circ} \mathrm{C}$ as the final extension. The amplified products were separated on 1\% agarose gel and a DNA marker of $100 \mathrm{bp}$ (DNA Ladder Plus, MBI Fermentas) was used to estimate the band size of each amplicon (Table 1). For visualization and photography, Gel documentation system (Gene Flash, Syngene Biolmaging, Vaughan, Canada) was used under UV light.

\subsection{Structural and functional analysis of selected marker}

According to the results of previous step, the amplicons of the marker $G B$ that clearly differentiated resistant genotypes from susceptible ones in both wheat and Ae. tauschii, were extracted and purified using a gel extraction kit (Fermentase, Germany) according to the manufacturer's protocol, and sequenced (Macrogen, South Korea). The sequences from Ae. tauschii and wheat were trimmed and assembled separately, using the CLC Genomics Workbench 9.5 software (https://digitalinsights.qiagen.com/products-overview/discovery-insights-portfolio/analysis-andvisualization/qiagen-clc-genomics-workbench/). Then, resultant contigs were aligned to find possible dissimilarities and were subsequently subjected to BLASTn against Ae. tauschii and wheat genomes at the International Wheat Genome Sequencing Consortium (IWGSC) (https://www.wheatgenome.org/) with E-values $\leq 10^{-5}$. All possible open reading frames (ORFs) of the best BLASTn hits were assigned with the same software, of which the longest ones were searched against NCBI Non-redundant protein sequences (nr) database by BLASTx (E-value $\leq 10^{-5}$ ). The amino acid sequences of the predicted ORFs were also specified and aligned using the CLC Genomics Workbench software 9.5. Protein domains of deduced protein sequences were identified using Pfam 34.0 (http://pfam.xfam.org/).

Subsequently, predicted ORFs (hereinafter referred to as the predicted genes) were mapped on the Ae. tauschii and wheat genome using IWGSC (https://www.wheatgenome.org/). The corresponding genomic regions containing $2.5 \mathrm{cM}$ upstream and downstream of the identified genes were selected. Therefore, up to $500 \mathrm{~Kb}$ genomic regions were retrieved from IWGSC, and all possible ORFs were predicted considering the following parameters:

- -Start codon; AUG,

- -Search; Both strands

- -Minimum length (codon);

- -250, Genetic code; Standard.

Then, all predicted ORFs were subjected to BLASTx search against the Arabidopsis Information Resource (TAIR) (https://www.arabidopsis.org/) and NCBI nr databases using the same software. Afterwards, all obtained protein codes from BLASTx were networked to a hierarchical gene network using the Pathway Studio 7 software (https://www.pathwaystudio.com), based on Fisher's exact test (P-value $\leq 0.05)$. A number of small molecules, functional groups and cell processes were also added to the predicted network to enrich its biological information. 


\section{Results}

\subsection{Phenotypic and genotypic evaluations}

The genotypes TcLr19 and Agatha, and the Ae. tauschii accessions SU442 and SU444 displayed IT '0;1' while the accessions SU350 showed IT $3^{+}$similar to that of Thatcher (Table 2). These ITs indicated the presence of at least one leaf rust resistance gene in accessions SU442 and SU444, with a similar IT to that of $L r 19$-carrying wheat genotypes. This putative gene was likely an orthologue for $L r 19$, and hence was named Ae. tauschii-Lr19 (AtLr19) hereafter and was further investigated.

Table 2

Leaf rust responses of wheat and Ae. tauschii genotypes to Puccninia triticina pathotype CDHLQ at the seedling stage.

\begin{tabular}{|c|c|c|c|c|}
\hline \multirow[t]{2}{*}{ No. } & \multirow{2}{*}{\multicolumn{2}{|c|}{ Genotype / Accessions }} & \multicolumn{2}{|c|}{ Leaf rust reaction } \\
\hline & & & Infection Type ${ }^{b}$ & response \\
\hline 1 & \multirow[t]{3}{*}{ T. aestivum } & Thacher $+L r 19$ & 0 & resistant \\
\hline 2 & & Agatha & ; & resistant \\
\hline 3 & & Thatcher & $3^{+}$ & susceptible \\
\hline 4 & \multirow[t]{3}{*}{ Ae. tauschii ${ }^{\mathrm{a}}$} & SU442 & ; & resistant \\
\hline 5 & & SU444 & 0 & resistant \\
\hline 6 & & SU350 & $3^{+}$ & susceptible \\
\hline
\end{tabular}

${ }^{a}$ Indicates Shiraz University accession number.

b Infection types were scored based on a modified scale (Mclntosh et al., 1995) in which '0' = no visible uredinia; ',' = hypersensitive flecks; ' 1 ' = small uredinia with necrosis; ' 2 ' = small to moderate size uredinia with green islands surrounded by necrosis or chlorosis; ' 3 ' $=$ moderate size uredinia with or without chlorosis; $\mathrm{C}$ = chlorosis; ' $\mathrm{N}$ ' = necrosis; ' $\mathrm{X}$ ' = Heterogeneous. '-' and '+' denote smaller or larger uredinia.

The molecular marker tests using the STS primer GB discriminated resistant and susceptible genotypes in wheat and Ae. tauschii, where a $130 \mathrm{bp}$ amplicon was detected in the resistant plants but no band was amplified in the susceptible ones. The SSR marker Xwmc221 detected a $200 \mathrm{bp}$ amplicon in TcLr19, Agatha, and the resistant Ae. tauschii accessions of SU442 and SU444 while a 220 bp band was present in Thatcher and the susceptible genotype of SU350 (Figure 1). The PCR products with two SCAR primers; SCS123 and SCS253 were monomorphic that showed bands of 680 and 750 bp length, respectively, in all resistant and susceptible genotypes except for TcLr19, where no band was amplified.

\subsection{Structural analysis of selected marker}


Since the $G B$ marker exclusively amplified bands in resistant individuals, the corresponding PCR products from TCLr19 and the resistant Ae. tauschii accession (SU444) were sequenced and subjected to complementary analysis to identify similarity/difference between the amplicons.

The sequenced forward (105 bp) and reverse (108 bp) fragments in Ae. tauschii were trimmed and assembled into a $130 \mathrm{bp}$ contig. Similarly, a $101 \mathrm{bp}$ of forward fragment and a $99 \mathrm{bp}$ of reverse fragment were assembled into a $130 \mathrm{bp}$ contig in hexaploid wheat. The two contigs had $93.85 \%$ similarity and only differed in positions 24, 32, 33, 68, 74, 78, 95 and 120 (Figure 2), which could represent potential SNPs. The BLASTn search (E-value $\leq 0.00001$ ) against the Ae. tauschii genome sequences in the IWGSC database revealed $94 \%$ homology between the obtained contig and contig No. 1016110 of 3380 bp length in the database.

All possible ORFs in the contig no. 1016110 were identified with CLC genomics workbench 9.5. Of them, the longest was $870 \mathrm{bp}$ and the only ORF that included at least one of the primers. This ORF includes the forward primer at the beginning of its 5 ' site and consequently, the obtained contig covered $40 \mathrm{bp}$ of the coding region and $90 \mathrm{bp}$ of $5^{\prime}$ UTR of the considered ORF (Figure 3. A). The reverse primer was completely matched while the forward primer differed with a single nucleotide change from $\mathrm{G}$ to $\mathrm{T}$. This resulted in generating a $130 \mathrm{bp}$ fragment, the same size as the PCR product and with a high homology between the PCR amplicon and the corresponding region in the Ae. tauschii genome.

The 130 bp contig of wheat had $90 \%$ similarity to a region on 7D chromosome based on the IWGSC BLASTn results. An ORF of 864 bp length was predicted in this region, which included forward primer at $5^{\prime}$ site with three nucleotide substitutions (Figure 3. B). As before, the reverse primer was located in $5^{\prime}$ UTR of the considered ORF with six substitutions (Figure 3. B). These nucleotide differences were predictable because the reference genome for BLASTn was Chinese spring, while the amplified sequence in this study was from a resistant wheat genotype. In total, the obtained contig covered $40 \mathrm{bp}$ of the coding region and $89 \mathrm{bp}$ of 5 ' UTR of the predicted ORF.

The results of BLASTx for the predicted ORFs in Ae. tauschii and wheat showed that both ORFs had a very high homology (up to 99\%) to a gene encoding O-methyltransferase-2 (OMT2, XP_020168265). Pfam search revealed that the deduced amino acid sequences of AtOMT2 (Ae. tauschii-OMT2) and TaOMT2 (T. aestivum- OMT2) encompassed two protein domains, i.e. Methyltransferase2 and Dimerization (Table 3). 
Table 3

Protein domains related to the selected ORFs in Ae. tauschii and T. aestivum, AtOMT2 and TaOMT2, determined in Pfam protein database.

\begin{tabular}{|c|c|c|c|c|c|c|}
\hline \multirow[t]{2}{*}{ Protein $^{a}$} & \multirow[t]{2}{*}{ Domain } & \multicolumn{2}{|c|}{ Situation (bp) } & \multirow[t]{2}{*}{ Accession } & \multirow[t]{2}{*}{ E-value } & \multirow[t]{2}{*}{ Description } \\
\hline & & Start & End & & & \\
\hline \multirow[t]{2}{*}{ AtOMT2 } & Methyltransf_2 & 125 & 277 & PF00891.15 & $1.20 \mathrm{E}-22$ & O-methyltransferase \\
\hline & Dimerization & 34 & 82 & PF08100.8 & $2.10 \mathrm{E}-10$ & Dimerization domain \\
\hline \multirow[t]{2}{*}{ TaOMT2 } & Methyltransf_2 & 125 & 278 & PF00891.15 & 3.60E-22 & O-methyltransferase \\
\hline & dimerization & 34 & 82 & PF08100.8 & 3.10E-10 & Dimerization domain \\
\hline
\end{tabular}

The predicted AtOMT2 and TaOMT2 genes were mapped on chromosome 7DL of Ae. tauschii and T. aestivum, respectively, with $100 \%$ identity. Up to $500 \mathrm{Kbp}$, including $250 \mathrm{Kbp}$ of each upstream and downstream of the predicted genes were retrieved in Ae. tauschii and wheat, separately. Selected regions were subjected to ORF finding to physically assign genes located near OMT2. Accordingly, 64 ORFs were assigned to Ae. tauschii. These ORFs had a minimum length of $759 \mathrm{bp}$, a maximum length of $3318 \mathrm{bp}$ and an average length of $1365 \mathrm{bp}$. BLASTx search showed that there are two ORFs related to $O$ methy/transferase-2, two disease resistance protein $R P M 1$-like, three disease resistance protein $R G A 2$ isoform X3, two hypothetical protein TRIUR3, one BURP12 domain-containing protein, and one ORF related to myosin-11 (Supplementary 1). Concerning wheat, 51 ORFs with a minimum length of $759 \mathrm{bp}$, a maximum length of $4326 \mathrm{bp}$ and an average length of $1468 \mathrm{bp}$ were predicted for the selected chromosomal region. BLASTx revealed that the majority of the predicted ORFs matched to unknown or putative proteins (Supplementary 2). Among the known proteins, five ORFs related to $O$ methyltransferase-2, one disease resistance protein RGA2 isoform $\mathrm{X} 3$, and four ORFs related to hypothetical protein TRIUR3 (Supplementary 2).

\subsection{Functional interpretation of predicted genes}

The predicted hierarchal gene network encompassed six identified putative genes, including OMT2, PR1, RPM1, ZAR1, MB-ARC and NBS-LRR. In addition, three genes, namely ICS1, ACS6 and PDF1.2, were added to the network by the Pathway Studio 7 software. The gene network also included small molecules, e.g. abscisic acid (ABA), ethylene (ET), salicylic acid (SA), jasmonic acid (JA), reactive oxygen species (ROS) and proline. Furthermore, five cellular processes, including programmed cell death (PCD), leaf senescence, stomata aperture, systemic acquired resistance (SAR) and necrosis, were included in the network nodes (Figure 4). Interestingly, the OMT2 and melatonin biosynthesis process were located upstream of all other nodes in the predicted gene network, emphasizing their importance.

\section{Discussion}


Phenotyping of wheat and Ae. tauschii genotypes supported the results of previous report by Aliakbari et al. (2020) as the resistant Ae. tauschii accessions SU442 and SU444 displayed low ITs similar to that of TcLr19 and Agatha. On contrary, the Ae. tauschii accession SU350 showed a high IT of $3^{+}$like the susceptible genotype Thatcher.

Molecular marker genotyping was used to verify the results of phenotyping where the dominant STS marker known as GB amplified a 130 bp fragment in the resistant genotypes and differentiated them from susceptible wheat and Ae. tauschii. Several studies have reported this marker as tightly linked with Lr19 that could identify it in diverse genotypic backgrounds (Gultyaeva et al., 2021; Pal et al., 2020; Prins et al., 2001). In another study, Maris (1992) reported its tight linkage with the water-soluble protein gene (WSP-D1) on 7DL. Afterwise, Prins et al. (2001) evaluated the AFLPs between Lr19 and WSP-D1 and converted a fragment to the GB marker. The SCAR markers SCS123 and SCS253 amplified bands in all genotypes except TcLr19, which produced no band. This clearly indicates that these markers are not suitable for screening the presence/absence of Lr19. These results are in agreement with those reported by Gupta et al. (2006) who stated that these markers did not amplify in TcLr19. Furthermore, the PCR amplification of codominant SSR marker Xwmc221 showed a fragment of 220 bp in the susceptible wheat and Ae. tauschii plants. In the isogeneic line TcLr19 and the cultivar Agatha, it produced a $200 \mathrm{bp}$ amplicon similar to that of SU442 and SU444 confirming the results of GB marker and was in agreement of previous studies by Gupta et al. (2006) and Gultyaeva et al. (2021).

Overall, the genotyping findings accompanied by the host-pathogen interaction indicated that the resistance gene present in Ae. tauschii could be similar to wheat Lr19. Since no other $L r$ gene except $L r 19$ has been reported on 7DL (Mclntosh et al., 2019), it becomes strongly evident that AtLr19 is an orthologue of Lr19. Orthologues of resistance in genotypes other than their original source are not unprecedented. In this context, Ling et al. (2004) identified and characterized an orthologue for $L r 1$ in $A e$. tauschii whereas this gene was first described in the hexaploid wheat (Ausemus et al., 1946). It is unknown whether this gene had evolved before or after hybridization of the AB genomes with the $D$ genome. It was speculated that $L r 1$ possibly evolved in Ae. tauschii and was later integrated into hexaploid wheat or the Lr1 might have evolved independently in Ae. tauschii and bread wheat (Ling et al., 2004).

The sequences of the amplified fragments from the GB marker in the resistant accession SU444 of $A e$. tauschii and TCLr19 were compared and bioinformatics analysis was performed to validate the results of phenotyping and genotyping. The results revealed that both sequences were the same and had more than 99\% homology confirming the presence of Lr19 orthologue (AtLr19) in Ae. tauschii. This segment is related to an independent gene responsible for encoding a methyltransferase member, $O$ methyltransferase-2 (OMT2). In plants, Methyltransferases constitute a large family of enzymes that methylate the oxygen atom of diverse secondary metabolites and play a key role in lignin biosynthesis, stress tolerance, and disease resistance (Lam et al., 2007). Increased accumulation of lignin can provide an elementary barrier against pathogen penetration into cell walls and consequently reduce the fungal toxins (Ma et al., 2017). 
Pfam search exhibited there are two protein domains Methyltransferase 2 and Dimerization in amino acid sequences of AtOMT2 and TaOMT2. In plants; Dimerization, contributes to the dimerization interface of the substrate binding and is critical for biological activities (Zubieta et al., 2001). Therefore, the presence of this domain in At-OMT2-ORF ascertains its involvement in functional in vivo and in vitro activities. Domain structures such as OMT2 and Dimerization in plants are involved in several functional metabolisms and consist of some domain-domain reactions, which lead to enhanced tolerance to various biotic and abiotic stresses. Among them, two domains Ank2 (Pf:12796) and F-box (Pf:00646) participate in protein structures along with OMT2 to induce resistance to pathogens through cell death and ABA signaling. The ANK protein family is a master regulator of the salicylic acid (SA) signaling pathway and functions as a transcriptional coactivator to regulate the plant defense response. Recent studies have indicated that some plant ANK family members confer resistance to rust diseases in wheat (Kolodziej et al., 2021; Wang et al., 2020). Likewise, F-box proteins are involved in abscisic acid (ABA) signaling that regulates cell death when the pathogen is recognized (van den Burg et al., 2008).

Anti-pathogenic mechanisms are characterized by the up-regulation of many defense genes. When a pathogen attacks, plants must perceive the signals and respond to the invasion in a timely manner (Jones and Dangl, 2006). As shown in pathway analysis, OMT2 is involved in melatonin biosynthesis as a master regulator molecule. Melatonin is an environmentally amicable molecule with multiple functions such as tolerance to biotic and abiotic stresses (Arnao and Hernández-Ruiz, 2018). The regulatory changes due to melatonin reinforce physiological, molecular processes, and hormone regulation. Moreover, melatonin acts as a super antioxidant against ROS and induces numerous changes in gene expression and signal transduction. The O-methylation of $\mathrm{N}$-acetylserotonin through an $\mathrm{O}$ methyltransferase reaction is believed to be the last step in melatonin biosynthesis (Kaur et al., 2015). It is presumed that pathogen's attack immediately causes melatonin to metabolize, and then melatonin participates in phytohormone signaling pathways to interfere with defense response (Moustafa-Farag et al., 2020). The collaboration of melatonin in upregulating genes such as plant resistance 1 (PR1), plant defensin1.2 (PDF1.2), an ethylene-related gene (ACS6), and Isochorismate synthase1 (ICS1) has been well studied (Zhao et al., 2021). Likewise, Sahu et al. (2021) reported that these genes are upregulated in rust resistant wheat under infected conditions. Altogether, the predicted gene network clearly illustrates the main pathways and components related to the OMT2, in the context of pathogen resistance.

Bioinformatics analysis of ORFs in contig no. 1016110 recognized two OMT2-related ORFs i.e. XP_020168264 and XP_020168265. These ORFs are located near the nucleotide region of the ORFs with biotic resistance responsibilities e.g., RPM1, RGA2, TRIUR3, BURP12 domain-containing protein, and myosin-11. The disease resistance protein RGA2 isoform X3 and the hypothetical protein TRIUR3 were common between the ORFs in Ae. tauschii and T. aestivum. These proteins play critical roles in biotic stress responses. The remaining ORFs belonged to different families of known or putative proteins. The $R P M 1$ and $R G A 2$ consist of disease-related domains, especially nucleotide-binding site leucine-rich repeat (NBS-LRR), coiled-coil (CC), and transmembrane (TM) like most R genes (Meyers et al., 2005). The gene Lr19 has an NBS-LRR structure (Gennaro et al., 2009) and as the domain components of RPM1 and RGA2 are the same as those of Lr19, one of these ORFs could be AtLr19. 
Wheat leaf rust continues to be a disease of concern in the majority of wheat-growing areas of the world. Perception of the molecular mechanisms governing defense signaling may provide a novel resistance mechanism for the sustainable management of rust diseases. Different molecular biology and bioinformatics tools have become instrumental for the analysis of transcriptional profiling, putative virulence gene identification, structural gene annotation, and alternative transcript splicing in host and pathogen. Based on the results of this study and previous reports by Aliakbari et al. (2020), it was speculated that an orthologue for $L r 19$ is responsible for leaf rust resistance in Ae. tauschii genotypes and hence was named AtLr19. Molecular and sequence analyses indicated high similarity in markerbased fragments in TcLr19 and Ae. tuaschii. Moreover, bioinformatics analysis revealed that the linked STS marker for Lr19 belongs to an O-methyltransferase 2 gene that is involved in some protein structures and gene pathways inducing disease resistance against fungi pathogens. AtLr19 might have evolved independently in Ae. tauschii or has been transferred to it by spontaneous outcrossing from $T h$. ponticum. It is the first report of finding Lr19 in Ae. tauschii and the results provide insights into resistance source and provide a framework for future studies aimed at the accurate dissection of AtLr19 and the genetic basis of OMT2 in resistance.

\section{Declarations}

The authors declare that they have no known competing financial interests or personal relationships that could have appeared to influence the work reported in this paper.

\section{Conflict of Interest}

The authors declare that there is no conflict of interest.

\section{Author contribution}

Zahra Pourkhorshid conducted the experiments, data analysis, and interpretation and drafted the manuscript; Ali Dadkhodaie designed the experiment, supervised the study, and revised the manuscript; Roohollah Shamloo-Dashtpagerdi contributed to the bioinformatics analysis and data interpretation and revised the manuscript.

\section{Availability of data and material}

Data and material will be available upon the request from the corresponding author if not deposited at public networks.

\section{References}

Aliakbari Sadeghabad, A., Dadkhodaie, A., Heidari, B., (2020). Phenotypic and genetic diversity of leaf rust resistance in wheat wild relatives. J. Phytopathol., 168(7-8), 428-438. https://doi.org/10.1111/jph.12907 
Arnao, M.B., Hernández-Ruiz, J., (2018). Melatonin and its relationship to plant hormones. Ann. Bot., 121(2), 195-207. https://doi.org/10.1093/aob/mcx114

Aslam, Z., Khattak, J. Z. K., Ahmed, M., \& Asif, M. (2017). A role of bioinformatics in agriculture. In Quantification of climate variability, adaptation and mitigation for agricultural sustainability (pp. 413434). Springer, Cham. https://doi.org/10.1007/978-3-319-32059-5_17

Ausemus, E.R., Harrington, J.B., Reitz, L.P., Worzella, W.W., (1946). A summary of genetic studies in hexaploid and tetraploid wheats. J Am Soc Agron., 38, 1082-1099.

Caetano-Anollés, G., (2005). Evolution of genome size in the grasses. Crop Sci., 45(5), 1809-1816. https://doi.org/10.2135/cropsci2004.0604

Feuillet, C., Langridge, P., Waugh, R., (2008). Cereal breeding takes a walk on the wild side. Trends Genet., 24(1), 24-32. https://doi.org/10.1016/j.tig.2007.11.001

Food and Agriculture Statistics., (2021). Food and agriculture organization of the United Nations. http://www.fao.org/food-agriculture-statistics/en.

Gennaro, A., Koebner, R.M., Ceoloni, C., (2009). A candidate for $L r 19$, an exotic gene conditioning leaf rust resistance in wheat. Funct. Integr., 9(3), 325-33. https://doi.org/10.1007/s10142-009-0115-1

Gultyaeva, E., Shaydayuk, E., Gannibal, P., (2021). Leaf rust resistance genes in wheat cultivars registered in Russia and their influence on adaptation processes in pathogen populations. Agriculture, 11(4), 319. https://doi.org/10.3390/agriculture11040319

Gupta, S.K., Charpe, A., Prabhu, K.V., Haque, Q.M.R., (2006). Identification and validation of molecular markers linked to the leaf rust resistance gene Lr19 in wheat. Theor. Appl. Genet., 113(6), 1027-1036. https://doi.org/10.1007/s00122-006-0362-7

Huerta-Espino, J., Singh, R.P., German, S., McCallum, B.D., et al., (2011). Global status of wheat leaf rust caused by Puccinia triticina. Euphytica, 179(1), 143-160. https://doi.org/10.1007/s10681-011-0361-x Jones, J.D. and Dangl, J.L., (2006). The plant immune system. Nature, 444(7117), 323-329. https://doi.org/10.1038/nri3141

Kaur, H., Mukherjee, S., Baluska, F., Bhatla, S.C., (2015). Regulatory roles of serotonin and melatonin in abiotic stress tolerance in plants. Plant Signal. Behav., 10(11).

https://doi.org/10.1080/15592324.2015.1049788

Kolodziej, M.C., Singla, J., Sánchez-Martín, J., Zbinden, H., et al., (2021). A membrane-bound ankyrin repeat protein confers race-specific leaf rust disease resistance in wheat. Nat. Commun., 12(1), 1-12. https://doi.org/10.1038/s41467-020-20777-x 
Lam, K.C., Ibrahim, R.K., Behdad, B., Dayanandan, S., (2007). Structure, function, and evolution of plant Omethyltransferases. Genome, 50(11), 1001-1013.https://doi.org/10.1139/G07-077

Ling, H.Q., Qiu, J., Singh, R.P., Keller, B., (2004). Identification and genetic characterization of an Aegilops tauschii ortholog of the wheat leaf rust disease resistance gene Lr1. Theor. Appl. Genet., 109(6), 11331138. https://doi.org/10.1007/s00122-004-1734-5

Ma, Q.H., Zhu, H.H., Han, J.Q., (2017). Wheat ROP proteins modulate defense response through lignin metabolism. Plant Sci., 262, 32-38. https://doi.org/10.1016/j.plantsci.2017.04.017

Marais, G.F., (1992). Gamma irradiation induced deletions in an alien chromosome segment of the wheat 'Indis' and their use in gene mapping. Genome, 35(2), 225-229. https://doi.org/10.1139/g92-034

McCALLUM, B.D., Reimer, E., McNABB, W.I.N.N.I.E., Foster, A., Rosa, S., Xue, A., (2021). Physiologic specialization of Puccinia triticina, the causal agent of wheat leaf rust, in Canada in 2015-2019. Can. J. Plant Pathol. https://doi.org/10.1080/07060661.2021.1888156

McIntosh, R.A., Dubcovsky, J., Rogers, W.J., Xia, X.C., Raupp, W.J., (2019). Morphological and physiological traits. Annual wheat newsletter, p.98.

Mclntosh, R.A., Wellings, C.R., Park, R.F., (1995). Wheat rusts: an atlas of resistance genes. CSIRO publishing.

Meyers, B.C., Kaushik, S., Nandety, R.S., (2005). Evolving disease resistance genes. Curr. Opin. Plant Biol., 8(2), 129-134.https://doi.org/10.1016/j.pbi.2005.01.002

Mondal, S., Rutkoski, J.E., Velu, G., Singh, P.K., et al., (2016). Harnessing diversity in wheat to enhance grain yield, climate resilience, disease and insect pest resistance and nutrition through conventional and modern breeding approaches. Front. Plant Sci., 7, 991. https://doi.org/10.3389/fpls.2016.00991

Moustafa-Farag, M., Almoneafy, A., Mahmoud, A., Elkelish, A., Arnao, M.B., Li, L., Ai, S., (2020). Melatonin and its protective role against biotic stress impacts on plants. Biomolecules, 10(1), 54.

https://doi.org/10.3390/biom10010054

Pal, D., Bhardwaj, S.C., Sharma, P., Sharma, D., Khan, H., Babu, H.P., et al., (2020). Molecular marker aided selection for developing rust resistant genotypes by pyramiding Lr19/Sr25 and $\mathrm{Yr} 15$ in wheat (Triticum aestivum L.). Australas. Plant Pathol., 49(6), 631-640. https://doi.org/10.1007/s13313-020-00738-0

Pardey, P.G., Beddow, J.M., Kriticos, D.J., Hurley, T.M., et al., (2013). Right-sizing stem-rust research. Science, 340(6129), 147-148. https://doi.org/10.1126/science.122970

Prins, R., Groenewald, J.Z., Marais, G.F., Snape, J.W., Koebner, R.M.D., (2001). AFLP and STS tagging of $L r 19$, a gene conferring resistance to leaf rust in wheat. Theor. Appl. Genet., 103(4), 618-624. https://doi.org/10.1007/PL00002918 
Randhawa, M., Bansal, U., Lillemo, M., Miah, H., Bariana, H., (2016). Postulation of rust resistance genes in Nordic spring wheat genotypes and identification of widely effective sources of resistance against the Australian rust flora. J. Appl. Genet., 57(4), 453-465. https://doi.org/10.1007/s13353-016-0345-6

Sahu, R., Prabhakaran, N., Kundu, P., Kumar, A., (2021). Differential response of phytohormone signalling network determines nonhost resistance in rice during wheat stem rust (Puccinia graminis f. sp. tritici) colonization. Plant Pathol., 70(6), 1409-1420. https://doi.org/10.1111/ppa.13376

Sarma, D., Knott, D.R., (1966). The transfer of leaf-rust resistance from Agropyron to Triticum by irradiation. Can J Genet Cytol., 8(1), 137-143. https://doi.org/10.1139/g66-018

Stormo, G.D., (2000). DNA binding sites: representation and discovery. Bioinform., 16(1), 16-23. https://doi.org/10.1093/bioinformatics/16.1.16

Toth, J., Pandurangan, S., Burt, A., Mitchell Fetch, J., Kumar, S., (2018). Marker-assisted breeding of hexaploid spring wheat in the Canadian prairies. Can. J. Plant Sci., 99(2), 111-127. https://doi.org/10.1139/cjps-2018-0183

Van den Burg, H.A., Tsitsigiannis, D.I., Rowland, O., Lo, J., Rallapalli, G., et al., (2008). The F-box protein ACRE189/ACIF1 regulates cell death and defense responses activated during pathogen recognition in tobacco and tomato. The Plant Cell, 20(3), 697-719. https://doi.org/10.1105/tpc.107.056978

Wang, F., Yuan, S., Wu, W., Yang, Y., Cui, Z., Wang, H., Liu, D., (2020). TaTLP1 interacts with TaPR1 to contribute to wheat defense responses to leaf rust fungus. PLoS Genet., 16(7), e1008713. https://doi.org/10.1371/journal.pgen.1008713

Wellings, C.R., (2011). Global status of stripe rust: a review of historical and current threats. Euphytica, 179(1), 129-141. https://doi.org/10.1007/s10681-011-0360-y

Yu, G., Hatta, A., Periyannan, S., Lagudah, E., Wulff, B.B., (2017). Isolation of wheat genomic DNA for gene mapping and cloning. In wheat rust diseases (pp. 207-213). Humana Press, New York, NY. https://doi.org/10.1007/978-1-4939-7249-4_18

Zhao, D., Wang, H., Chen, S., Yu, D., Reiter, R.J., (2021). Phytomelatonin: an emerging regulator of plant biotic stress resistance. Trends Plant Sci., 26(1), 70-82. https://doi.org/10.1016/j.tplants.2020.08.009

Zubieta, C., He, X.Z., Dixon, R.A., Noel, J.P., (2001). Structures of two natural product methyltransferases reveal the basis for substrate specificity in plant O-methyltransferases. Nat. Struct. Biol., 8(3), 271-279. https://doi.org/10.1038/85029

\section{Figures 1-4}

Figures 1-4 were not provided with this version. 


\section{Supplementary Files 1-2}

Supplementary Files 1-2 were not provided with this version.

Supplementary 1. The BLASTx search results for ORFs were subjected to obtain physically assign genes located near OMT2 and their protein codes in Ae. tauschii.

Supplementary 2. The BLASTx search results for ORFs were subjected to obtain physically assign genes located near OMT2 and their protein codes in T. aestivum. 